\title{
6x scale-up while maintaining stable production of $n$-caprylic acid
}

\author{
Byoung Seung Jeon ${ }^{*}$,, Kurt Gemeinhardt ${ }^{\mathrm{a}}$, and Largus T. \\ Angenent $^{a}$ \\ * Presenter, Dr. Byoung Seung Jeon \\ a Environmental Biotechnology Group, Center for Applied Geosciences, \\ University of Tübingen, Schnarrenbergstraße 94-96, 72076, Tübingen, \\ Germany.
}

\section{HIGHLIGHTS:}

- $n$-Caprylic acid has certain advantages compared to $n$-caproic acid, including a reduced odour and a higher bactericidal activity.

- Stable production of $n$-caprylic acid was possible with a 4.2-L wet volume bioreactor that included pertraction, which is a 6x scale-up from our previous work at Cornell University (1).

- Stable production occurred at an ethanol-to-acetate substrate ratio of $6: 1$.

\section{BACKGROUND:}

To increase the product portfolio of microbial chain elongation, we are developing a stable bioprocess system with membrane-based liquidliquid extraction (pertraction) to include $n$-caprylic acid (C8; $n$-octanoic acid) in addition to other medium-chain carboxylic acids (MCCAs) such as n-caproic acid (C6; $n$-hexanoic acid) and $n$-heptanoic acid (C7). Certain advantages of $\mathrm{C} 8$ compared to $\mathrm{C} 6$ exist. This includes a higher bactericidal activity, a higher heat capacity (297.9 J/K mol), a 10x lower maximum solubility concentration, and a less unpleasant odour. At Cornell University, we had already achieved a C8-to-C6 productivity ratio of more than 20:1 by feeding a mixture of ethanol and acetate into an anaerobic filter (AF) as an open-culture system (1). However, this had been accomplished with a relatively small wet volume of $0.7 \mathrm{~L}$. Here, we scaled up the process $6 \mathrm{x}$ to a 4.2-L wet volume AF. We investigated whether we could repeat this result from Cornell University with a stable production of a considerably higher amount of C8 than C6 when a mixture of ethanol and acetate was fed as substrate. Such substrate mixture is present in the effluent of syngas fermentation systems.

\section{RESULTS \& DISCUSSION :}

The C8-producing AF was filled with K1-filter media (Kaldnes) in a 5$L$ glass upflow bioreactor with an active volume of 4.2 in the presence of the filter media. The fermentation broth was recirculated continuously through a forward membrane contactor together with a solvent as part of 
the pertraction system. Continuous extraction of MCCAs was achieved by recirculating this solvent and an alkaline stripping solution through a backward membrane contactor. We verified the molecular structure of the produced $\mathrm{C} 8$ through GC/MS. In addition, we monitored the produced metabolites by GC/FID. The C8 production performance was evaluated within an experimental design by changing the substrate ratio of ethanol to acetate. When the substrate ratio of ethanol and acetate was $6: 1$, the C8-to-C6 productivity ratio was higher than $2.5: 1$. This can be seen by the $\sim 2.5 \mathrm{x}$ steeper slope of the increase in the concentration of C8 compared to $\mathrm{C} 6$ in the alkaline stripping solution (Figure 1A), and also by the productivity ratio bar (Figure 1B). However, when the substrate ratio of ethanol to acetate was increased to $10: 1$, the overall C8-to-C6 productivity ratio decreased to $\sim 1.5: 1$ due to lower production of $\mathrm{C} 8$, while the production of C6 remained constant (Figure 1B). By reversing the substrate ratio back to $6: 1$, we again achieved a stable production of $\mathrm{C} 8$, which was $\sim 2.5 \mathrm{x}$ higher than $\mathrm{C} 6$ at a $6: 1$ substrate ratio of ethanol and acetate. Nevertheless, we have not been able to achieve the C8-toC6 productivity ratio of 20:1, which we observed at Cornell University, and we are now trying to understand why.

\section{CONCLUSION:}

The bioreactor system for this study accumulated C8 up to $8000 \mathrm{mM}$ $C$ in the stripping solution (Figure 1A). We achieved a stable production of $\mathrm{C} 8$ at a $6: 1$ substrate ratio of ethanol to acetate. 


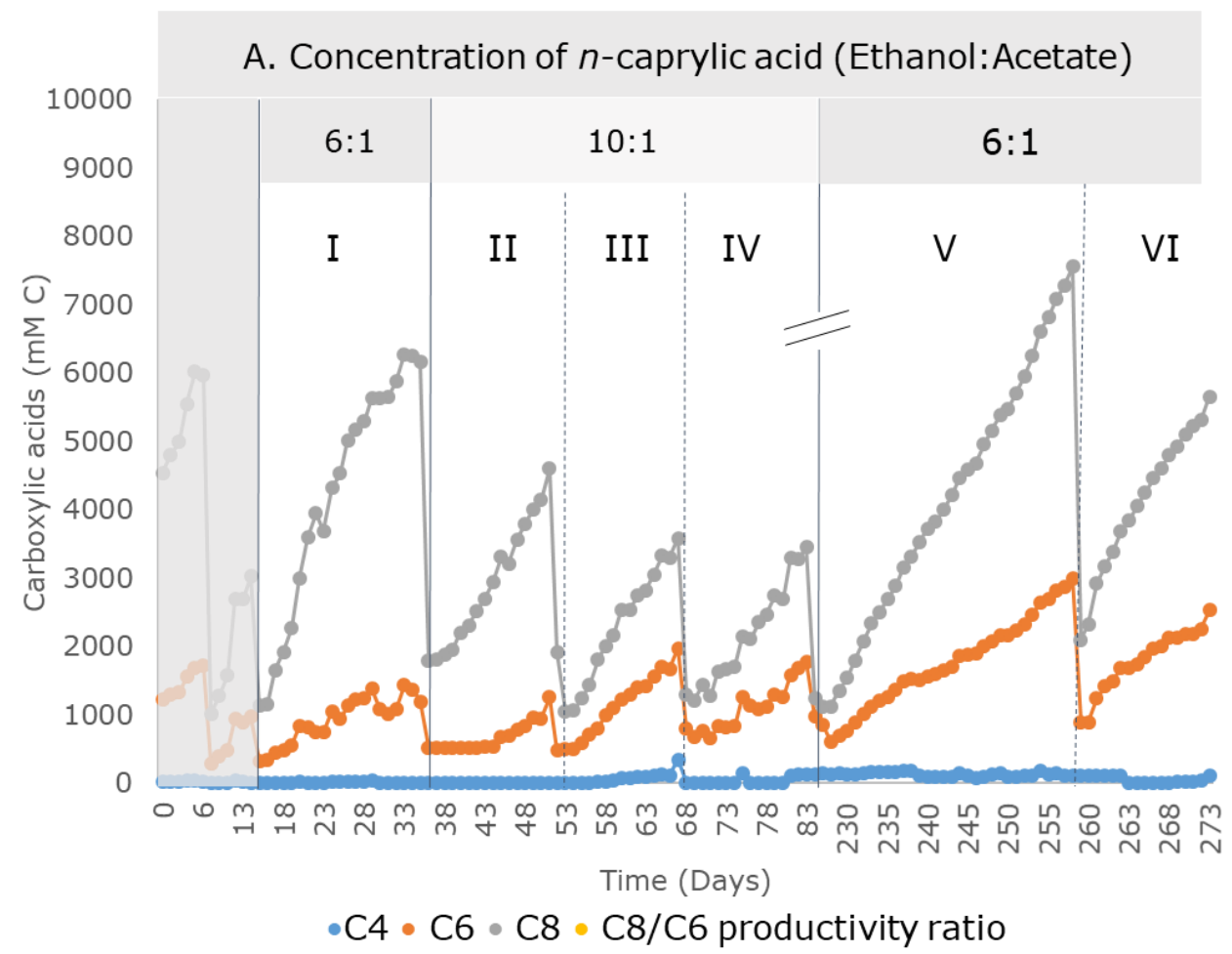

B. Average productivity and the $\mathrm{C} 8$-to- $\mathrm{C} 6$ productivity ratio

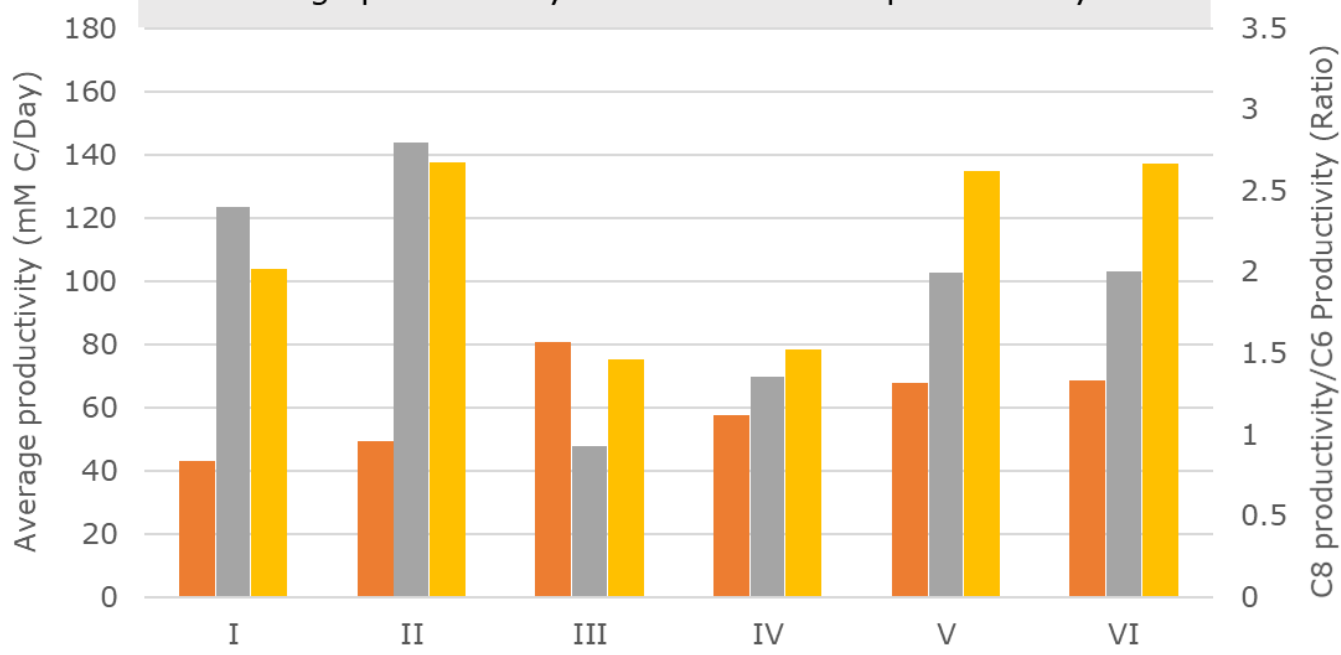

Figure 1: A. Concentration of $n$-caprylic acid in the alkaline stripping solution during the operating period (after each period the stripping solution was exchanged); productivity ratio.

\section{REFERENCES}

1. Leo A. Kucek, Catherine M. Spirito and Largus T. Angenent. High n-caprylate productivities and specificities from dilute ethanol and acetate: chain elongation with microbiomes to upgrade products from syngas fermentation. Energy \& Environmental Science, $2016,9,3482-3494$ 